\title{
A Unified Approach for Probability of \\ Detection Evaluation over Generalised Fading
}

\section{Channels}

\author{
Moataz M. H. El Ayadi ${ }^{1}$, Mahmoud H. Ismail ${ }^{2,3}$, and Husam R. Alhennawi ${ }^{3}$ \\ ${ }^{1}$ Department of Engineering Mathematics and Physics, Faculty of Engineering, \\ Cairo University, Giza 12613, Egypt. Email: moataz@eng.cu.edu.eg. \\ ${ }^{2}$ Department of Electrical Engineering, American University of Sharjah, PO Box \\ 26666, Sharjah, UAE. Email: mhibrahim@aus.edu. \\ ${ }^{3}$ Department of Electronics and Electrical Communications Engineering, Faculty \\ of Engineering, Cairo University, Giza 12613, Egypt
}

\begin{abstract}
In this paper, we revisit energy detection-based spectrum sensing cognitive radio systems operating over generalised fading channels. In particular, we derive closed-form exact expressions as well as lowand high-signal-to-noise ratio asymptotic expansions for the misdetection probability over the Fox's $H$-function fading channel. The closed-form expression is given in terms of the bivariate Fox's $H$ function and subsumes most of the expressions previously presented in the literature. Also, the obtained asymptotic expressions are very easy to compute and can be used to get various performance insights. We verified, theoretically and numerically, the validity of the exact expression for important special cases previously reported in the literature, namely the Nakagami- $m$ and the extended generalised- $K$ (EGK) fading distributions. Numerical results also demonstrate the high accuracy of the asymptotic expansions.
\end{abstract}

Keywords. Energy detection, cognitive radios, spectrum sensing, Fox's $H$-fading. 


\section{INTRODUCTION}

Among the different signal detection schemes, energy detection (ED) has stood out due to its simplicity and ability to sense the unknown signal without any prior knowledge about its characteristics nor about the channel gain [1]. The main idea of ED is comparing the received energy over an observation window with a predefined threshold and then deciding whether a specific signal exists or not. While ED is preferable over other detection schemes, it is severely affected by fading and its performance becomes poor at low signal-to-noise ratios (SNRs).

With the advent of cognitive radio systems as a possible solution for the spectrum scarcity problem, lots of works have been devoted to analyze the performance of ED spectrum sensing when operating over different fading models. The classical way for obtaining the average probability of detection over a specific fading distribution is through averaging the probability of detection of the additive white Gaussian noise (AWGN) channel over the probability density function (PDF) of the output SNR (e.g., [2]). This approach works well with many fading models [1], [3], [4]. However, with more complicated fading models, the resulting integrals become prohibitively complicated and rarely result in closed-form results. Recently, a new approach has been presented in [5] for analyzing the performance of ED. This approach is based on using the contour integral representation of the Marcum- $Q$ function along with the moment generating function (MGF) of the SNR. Results pertaining to Rician and Nakagami- $m$ fading were then presented but in terms of infinite series. Beside using the MGF approach with the classical fading models, it has also been used with more generalised fading models in [6], [7], [8], [9], [10], [11] and [12].

From the above discussion, it is clear that the literature lacks a unified approach that can be used to obtain the probability of detection over the ever-increasing number of fading channel models. Towards that end, in this paper, we derive a novel closed-form generalised expression and asymptotic expansions for the probability of misdetection over Fox's $H$-fading. The Fox's $H$-distribution has been very recently used as a generalised fading model [13]. In [14], the authors showed that it actually models fading in vehicle-to-vehicle (V2V) communication better than other ordinary fading distributions. Our derivation is based on the framework we developed previously in [15] for calculating the symbol error rate (SER) over fading channels. The probability of misdetection can be expressed in the form of a double Mellin-Barnes integral, which can 
then be transformed into a bivariate Fox's $H$-function. To the best of our knowledge, the resulting expression is the most generalised and unified form, which can handle the average probability of detection over most of the well-known fading distributions. The average probability of detection over two special cases of the Fox's $H$-fading are then discussed, namely, the Nakagami- $m$ and the EGK. Similar to our previous work in $[15,16]$, we also derive asymptotic expansions of the misdetection probability for large and small values of the average SNR.

The rest of the paper is organized as follows. The unified approach for the probability of misdetection computation is presented in the following section. It is then applied to the case of $H$-function fading in Section III where special cases are also considered and shown to reduce to those previously studied in the literature. Asymptotic expansions at low and high average SNRs are addressed in section IV. Numerical results are then presented and discussed in Section V before the paper is finally concluded in Section VI.

\section{The Average Probability of Detection: A Unified Approach}

The conventional method of evaluating $\bar{P}_{d}$ over fading channels is through

$$
\bar{P}_{d}=\int_{0}^{\infty} P_{d}(\gamma) f_{\gamma}(\gamma) d \gamma
$$

For the AWGN channel, $P_{d}(\gamma)$, has been derived in [1, Eq. (5)] and is given by $P_{d}(\gamma)=$

$Q_{u}(\sqrt{2 \gamma}, \sqrt{\lambda})$ where $u=W T, W$ is the one-sided bandwidth, $T$ is the observation time and $\lambda$ is the threshold used in ED, which can be selected based on the required probability of false alarm $P_{f}$ according to $P_{f}=\Gamma(u, \lambda / 2) / \Gamma(u)$ where $\Gamma(.,$.$) is the upper incomplete Gamma$ function. Also, $Q_{u}($.$) is the generalised Marcum Q$-function, defined by [17, Eq. (4.74)]

$$
Q_{u}(\alpha, \beta)=\frac{1}{\alpha^{u-1}} \int_{\beta}^{\infty} x^{u} \exp \left(-\frac{x^{2}+\alpha^{2}}{2}\right) I_{u-1}(\alpha x) d x,
$$

where $I_{k}(\cdot)$ is the $k$ th-order modified Bessel function of the first kind. It is actually more convenient to derive a closed-form expression for the misdetection probability, $\bar{P}_{m}=1-\bar{P}_{d}$ since $\lim _{\gamma \rightarrow \infty} P_{d}(\gamma)=1$ and hence, $P_{d}(\gamma)$ does not have a Mellin transform, which will be the primary tool of our analysis. It is straightforward to show that the $\bar{P}_{m}$ is given by

$$
\bar{P}_{m}=\int_{0}^{\infty} P_{m}(\gamma) f_{\gamma}(\gamma) d \gamma
$$


where $P_{m}(\gamma)=1-P_{d}(\gamma)$. Using the Parseval's relation for the Mellin transform [18, Eq. (2.31)], the misdetection probability is given by

$$
\bar{P}_{m}=\frac{1}{2 \pi i} \int_{s=\sigma-i \infty}^{\sigma+i \infty} P_{m}^{*}(1-s) f^{*}(s) d s,
$$

where $P_{m}^{*}(s)$ and $f^{*}(s)$ are the Mellin transforms of $P_{m}(\gamma)$ and $f_{\gamma}(\gamma)$, respectively, and $\sigma$ is a real constant, which lies in the domain of convergence (DOC) of both $f^{*}(s)$ and $P_{m}^{*}(1-s)$. Fortunately, the Mellin transform of $f_{\gamma}(\gamma)$ can be straightforwardly derived for the $H$-function and its special cases. Meanwhile, the Mellin transform of $P_{m}(\gamma)$ is given in the following theorem.

Theorem 1. If $0<\lambda<\infty$, the Mellin transform of $P_{m}(\gamma)$ is given by

$$
P_{m}^{*}(z)=\frac{1}{2 \pi i} \frac{\Gamma(z)}{\Gamma(u-z)} \int_{w=\sigma_{2}-i \infty}^{\sigma_{2}+i \infty} \frac{\Gamma(-w) \Gamma(u-z+w)}{\Gamma(u+1+w)}\left(\frac{\lambda}{2}\right)^{u+w} d w, \quad 0<\Re\{z\}<u,
$$

where $\sigma_{2}$ is a real constant such that $\Re\{z\}-u<\sigma_{2}<0$.

Proof. Starting with $P_{m}(\gamma)=1-Q_{u}(\sqrt{2 \gamma}, \sqrt{\lambda})$ and substituting $\alpha=\sqrt{2 \gamma}$ and $\beta=\sqrt{\lambda}$ in (2), we arrive at

$$
P_{m}(\gamma)=(2 \gamma)^{-(1-u) / 2} e^{-\gamma} \int_{0}^{\sqrt{\lambda}} x^{u} e^{-x^{2} / 2} I_{u-1}(x \sqrt{2 \gamma}) d x
$$

Hence, we have

$$
\begin{aligned}
P_{m}^{*}(z) & =\int_{\gamma=0}^{\infty} P_{m}(\gamma) \gamma^{z-1} d \gamma=\int_{\gamma=0}^{\infty} 2^{-(u-1) / 2} \gamma^{z-(u+1) / 2} e^{-\gamma} \int_{x=0}^{\sqrt{\lambda}} x^{u} e^{-x^{2} / 2} I_{u-1}(x \sqrt{2 \gamma}) d x d \gamma \\
& =\int_{x=0}^{\sqrt{\lambda}} x^{u} e^{-x^{2} / 2} \int_{\gamma=0}^{\infty} 2^{-(u-1) / 2} \gamma^{z-(1+u) / 2} e^{-\gamma} I_{u-1}(x \sqrt{2 \gamma}) d \gamma d x
\end{aligned}
$$

Using [19, Eq. (6.643.2)] and [19, Eq. (9.220.3)], the inner integral in the previous expression converges only for $\Re\{z\}>0$ and it reduces to $(\Gamma(z) / \Gamma(u))(x / 2)^{u-1}{ }_{1} F_{1}\left(z ; u ; x^{2} / 2\right)$ where ${ }_{1} F_{1}(. ; . ;$.$) is the confluent hypergeometric function. Consequently,$

$$
\begin{aligned}
P_{m}^{*}(z) & =\frac{\Gamma(z)}{\Gamma(u)} \int_{x=0}^{\sqrt{\lambda}} 2^{1-u} x^{2 u-1} e^{-x^{2} / 2}{ }_{1} F_{1}\left(z ; u ; x^{2} / 2\right) d x \\
& =\frac{\Gamma(z)}{\Gamma(u-z)} \int_{x=0}^{\sqrt{\lambda}} 2^{1-u} x^{2 u-1} \frac{1}{2 \pi i} \int_{w=\sigma_{2}-i \infty}^{\sigma_{2}+i \infty} \frac{\Gamma(-w) \Gamma(u-z+w)}{\Gamma(u+w)}\left(\frac{x^{2}}{2}\right)^{w} d w d t,
\end{aligned}
$$

in which we used the Mellin-Barnes definition of the confluent hypergeometric function [20, Eq. 13.4.16] in deriving (8b). Note that the Mellin-Barnes integral in (8b) is valid only if $\Re\{z\}<u$. 
Moreover, $\sigma_{2}$ must be chosen such that $\Re\{z\}-u<\sigma_{2}<0$. Interchanging the order of integrations in $(8 b)$ and performing the integration with respect to $x$, we easily derive the required result as long as $\lambda<\infty$.

Based on Theorem 1, substituting from (5) into (4), we derive the following final expression for $\bar{P}_{m}$.

$$
\bar{P}_{m}=\frac{1}{(2 \pi i)^{2}} \int_{s=\sigma-i \infty}^{\sigma+i \infty} \int_{w=\sigma_{2}-i \infty}^{\sigma_{2}+i \infty} \frac{\Gamma(1-s)}{\Gamma(u-s+1)} \frac{\Gamma(-w) \Gamma(u-s+1+w)}{\Gamma(u+1+w)}\left(\frac{\lambda}{2}\right)^{u+w} d w f^{*}(s) d s,
$$

where $\sigma$ lies in the intersection of the DOC of $f^{*}(s)$ and the interval $] 1-u, 1\left[\right.$. In addition, $\sigma_{2}$ must satisfy that $1-\Re\{s\}-u<\sigma_{2}<0$. The above expression is the main formula that will be used in deriving $\bar{P}_{m}$ in this work.

\section{Application to the Fox's $H$-Function Fading Channel}

The Fox's $H$-function is a general fading distribution whose PDF is defined by [21]

$$
f_{\gamma}(\gamma)=\mathcal{K} H_{p, q}^{m, n}\left(C \gamma \mid \begin{array}{l}
\left(a_{j}, A_{j}\right)_{j=1: p} \\
\left(b_{j}, B_{j}\right)_{j=1: q}
\end{array}\right)
$$

where the constants $C>0$ and $\mathcal{K}>0$ depend on the average SNR $\bar{\gamma}$ and are chosen such that $\int_{\gamma=0}^{\infty} f_{\gamma}(\gamma) d \gamma=1$. Using the definition of the $H$-function in [21], the Mellin transform of $f_{\gamma}(\gamma)$ is directly obtained as

$$
f^{*}(s)=\mathcal{K} C^{-s} \frac{\left.\prod_{j=1}^{m} \Gamma\left(b_{j}+B_{j} s\right) \prod_{j=1}^{n} \Gamma\left(1-a_{j}-A_{j} s\right)\right]}{\prod_{j=m+1}^{q} \Gamma\left(1-b_{j}-B_{j} s\right) \prod_{j=n+1}^{p} \Gamma\left(a_{j}+A_{j} s\right)} .
$$

A closed-form expression for the probability of misdetection can now be obtained by substituting (11) and (5) into (4) yielding

$$
\bar{P}_{m}=\frac{\mathcal{K}\left(\frac{\lambda}{2}\right)^{u}}{(2 \pi i)^{2}} \int_{s=\sigma_{1}-i \infty}^{\sigma_{1}+i \infty} \int_{w=\sigma_{2}-i \infty}^{\sigma_{2}+i \infty} \Xi(s) \frac{\Gamma(1-s) \Gamma(-w) \Gamma(u-1+s+w)}{\Gamma(u+s-1) \Gamma(u+1+w)}\left(\frac{2}{\lambda}\right)^{-w} C^{-s} d w d s,
$$

where

$$
\Xi(s)=\frac{\prod_{j=1}^{m} \Gamma\left(b_{j}+B_{j} s\right) \prod_{j=1}^{n} \Gamma\left(1-a_{j}-A_{j} s\right)}{\prod_{j=m+1}^{q} \Gamma\left(1-b_{j}-B_{j} s\right) \prod_{j=n+1}^{p} \Gamma\left(a_{j}+A_{j} s\right)},
$$

$\sigma_{1}$ and $\sigma_{2}$ are real constants such that $\max \left(-\min _{j=1, \ldots, m}\left(\Re\left\{\frac{b_{j}}{B_{j}}\right\}\right), 1-u\right)<\sigma_{1}<1$ and $1-\sigma_{1}-u<\sigma_{2}<0$. Often, it is desired to have the multiplicative constant proportional to the 
ratio $\mathcal{K} / C$ because it always depends on the parameters of the fading distribution only. This can be achieved by performing the change of variables $s=s^{\prime}+1$ in the previous expression and using the definition of the bivariate Fox's $H$-function [21] to obtain $\bar{P}_{m}=\frac{\mathcal{K}}{C}\left(\frac{\lambda}{2}\right)^{u} \times$

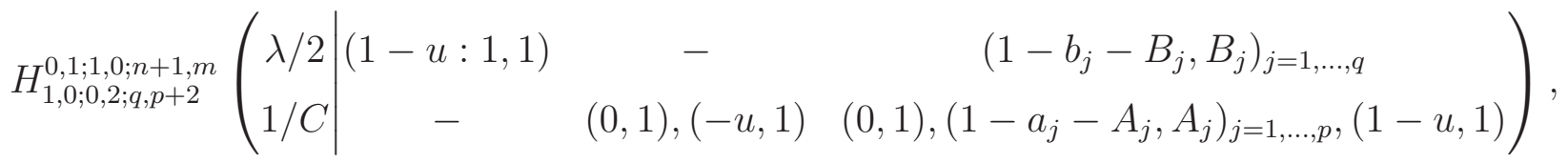

which represents a closed-form expression for the probability of misdetection. It is worth mentioning here that efficient implementations of the bivariate $H$-function using Python and MATLAB have already been presented in [16, 22], respectively. To the best of the authors' knowledge, (14) is the most general expression for $\bar{P}_{m}$ ever presented in the literature. It subsumes most, if not all, of the expressions previously presented for different fading distributions. In Table I, we state some common fading distributions together with their corresponding expressions for $\bar{P}_{m}$ as special cases of (14). In Appendices A and B, we prove the equivalence of our derived expressions for the cases of Nakagami- $m$ and EGK distributions to those previously found in the literature.

It is important to note that although the expression in (14) is very general, its evaluation, in fact, requires numerical integration, which limits its practical value. Moreover, when the value of $\bar{P}_{m}$ is very small, the evaluation is subject to numerical underflow, which adds another difficulty in evaluating the integral. Therefore, we present asymptotic expansions for $\bar{P}_{m}$ when $\bar{\gamma}$ is either very large $(\geq 20 \mathrm{~dB})$ or very low $(\leq 0 \mathrm{~dB})$ in the next section.

\section{Asymptotic Expansions for the Probability of Misdetection}

The main idea for deriving the asymptotic expansions is to represent the integration in (12) as a single integral in $s$ and evaluating the residues at the most significant poles of the integrand [23]. Towards that end, we note that the inner integral w.r.t. $w$ is a Mellin-Barnes representation of the confluent hypergeometric function [19]. Hence, $\bar{P}_{m}$ can be written as

$$
\bar{P}_{m}=\frac{\mathcal{K}\left(\frac{\lambda}{2}\right)^{u}}{2 \pi i} \int_{s=\sigma_{1}-i \infty}^{\sigma_{1}+i \infty} \Xi(s) \frac{\Gamma(1-s)}{\Gamma(u+1)}{ }_{1} F_{1}\left(s+u-1 ; u+1 ;-\frac{\lambda}{2}\right) C^{-s} d s .
$$


TABLE I

EXPRESSIONS OF $\bar{P}_{m}$ FOR DIFFERENT SPECIAL CASES OF THE FOX's $H$-FUnCTION Distribution.

\begin{tabular}{|c|c|}
\hline Fading distribution & $f_{\gamma}(\gamma)$ and $\bar{P}_{m}$ \\
\hline Rayleigh & $\begin{array}{c}f_{\gamma}(\gamma)=\frac{1}{\bar{\gamma}} \exp \left(-\frac{\gamma}{\bar{\gamma}}\right)=\frac{1}{\bar{\gamma}} H_{0,1}^{1,0}\left(\frac{\gamma}{\bar{\gamma}} \mid \begin{array}{c}- \\
(0,1)\end{array}\right) \\
\bar{P}_{m}=\left(\frac{\lambda}{2}\right)^{u} H_{1,0 ; 0,2 ; 1,2}^{0,1 ; 1,0 ; 1,1}\left(\begin{array}{cccc}\lambda / 2 & (1-u: 1,1) & - & (0,1) \\
\bar{\gamma} & - & (0,1),(-u, 1) & (0,1),(1-u, 1)\end{array}\right)\end{array}$ \\
\hline Maxwell & $\begin{array}{c}f_{\gamma}(\gamma)=\sqrt{\frac{27}{2 \pi \bar{\gamma}^{3}}} \exp \left(-\frac{3 \gamma}{2 \bar{\gamma}}\right)=\frac{3}{\sqrt{\pi} \bar{\gamma}} H_{0,1}^{1,0}\left(\frac{3 \gamma}{2 \bar{\gamma}} \mid \begin{array}{c}\left(\frac{1}{2}, 1\right)\end{array}\right) \\
\bar{P}_{m}=\frac{1}{\sqrt{\pi}}\left(\frac{\lambda}{2}\right)^{u} H_{1,0 ; 0,2 ; 1,2}^{0,1 ; 1,1 ; 1,1}\left(\begin{array}{c|ccc}\lambda / 2 & (1-u: 1,1) & - & \left(\frac{1}{2}, 1\right) \\
2 \bar{\gamma} & - & (0,1),(-u, 1) & (0,1),(1-u, 1)\end{array}\right)\end{array}$ \\
\hline Nakagami- $m$ & $\begin{array}{c}f_{\gamma}(\gamma)=\frac{1}{\Gamma(m)}\left(\frac{m}{\bar{\gamma}}\right)^{m} \gamma^{m-1} \exp \left(-m \frac{\gamma}{\bar{\gamma}}\right)=\frac{m}{\Gamma(m) \bar{\gamma}} H_{0,1}^{1,0}\left(\frac{m \gamma}{\bar{\gamma}} \mid \begin{array}{c}- \\
(m-1,1)\end{array}\right) \\
\bar{P}_{m}=\frac{1}{\Gamma(m)}\left(\frac{\lambda}{2}\right)^{u} H_{1,0 ; 0,2 ; 1,2}^{0,1 ; 1,0 ; 1,}\left(\begin{array}{c}\lambda / 2 \\
\bar{\gamma} / m\end{array} \mid \begin{array}{ccc}(1-u: 1,1) & - & (1-m, 1) \\
- & (0,1),(-u, 1) & (0,1),(1-u, 1)\end{array}\right)\end{array}$ \\
\hline Weibull & $\begin{array}{c}f_{\gamma}(\gamma)=\alpha\left(\frac{\beta}{\bar{\gamma}}\right)^{\alpha} \gamma^{\alpha-1} \exp \left(-\left(\frac{\beta \gamma}{\bar{\gamma}}\right)^{\alpha}\right)=\frac{\beta}{\bar{\gamma}} H_{0,1}^{1,0}\left(\frac{\beta \gamma}{\bar{\gamma}} \mid \begin{array}{c}- \\
\left(1-\frac{1}{\alpha}, \frac{1}{\alpha}\right)\end{array}\right), \quad \beta=\Gamma\left(1+\frac{1}{\alpha}\right) \\
\bar{P}_{m}=\left(\frac{\lambda}{2}\right)^{u} H_{1,0 ; 0,2 ; 1,2}^{0,1 ; 1,0 ; 1,1}\left(\begin{array}{c}\lambda / 2 \\
\bar{\gamma} / \beta\end{array} \mid \begin{array}{ccc}(1-u: 1,1) & - & \left(0, \frac{1}{\alpha}\right) \\
- & (0,1),(-u, 1) & (0,1),(1-u, 1)\end{array}\right)\end{array}$ \\
\hline Generalised Gamma & $\begin{array}{r}f_{\gamma}(\gamma)=\frac{\xi}{\Gamma(\mu)}\left(\frac{\beta}{\bar{\gamma}}\right)^{\xi \mu} \gamma^{\xi \mu-1} \exp \left(-\left(\frac{\beta \gamma}{\bar{\gamma}}\right)^{\xi}\right)=\frac{\beta}{\Gamma(\mu) \bar{\gamma}} H_{0,1}^{1,0}\left(\frac{\beta \gamma}{\bar{\gamma}} \mid \begin{array}{c}- \\
\left(\mu-\frac{1}{\xi}, \frac{1}{\xi}\right)\end{array}\right), \quad \beta=\frac{\Gamma\left(\mu+\frac{1}{\xi}\right)}{\Gamma(\mu)} \\
\bar{P}_{m}=\frac{1}{\Gamma(\mu)}\left(\frac{\lambda}{2}\right)^{u} H_{1,0 ; 0,2 ; 1,2}^{0,1 ; 1,0 ; 1,1}\left(\begin{array}{c}\lambda / 2 \\
\bar{\gamma} / \beta\end{array} \mid \begin{array}{ccc}(1-u: 1,1) & - & \left(1-\mu, \frac{1}{\xi}\right) \\
- & (0,1),(-u, 1) & (0,1),(1-u, 1)\end{array}\right)\end{array}$ \\
\hline EGK & 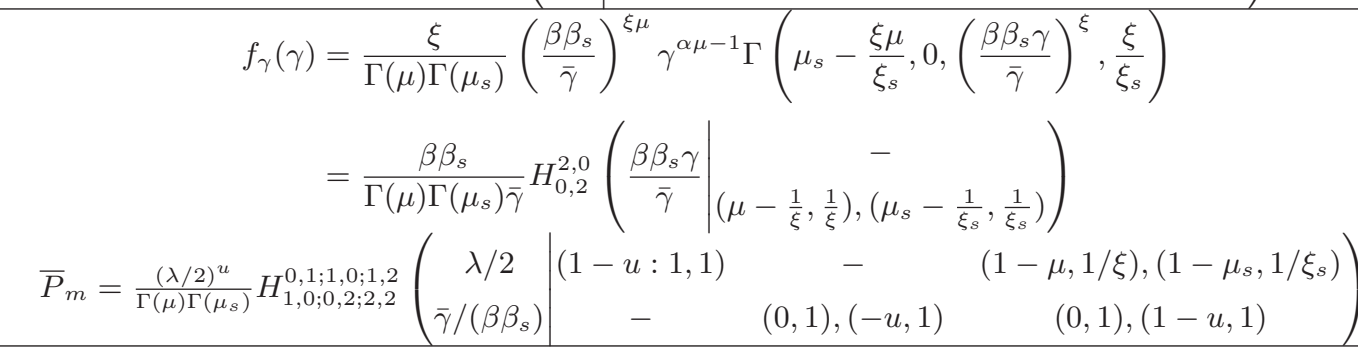 \\
\hline
\end{tabular}

The confluent hypergeometric function is an entire function, i.e., it does not impose any pole. Hence, we have three sets of poles in the integrand of (15): the poles of the factors $\Gamma\left(b_{j}+B_{j} s\right)$, which are given by $s=-\left(b_{j}+k\right) / B_{j}$ for $j=1, \ldots, m$ where $k \in\{0,1,2, \ldots\}$, the poles of the factors $\Gamma\left(1-a_{j}-A_{j} s\right)$, which are given by $s=\left(1-a_{j}+k\right) / A_{j}$ for $j=1, \ldots, n$ where $k \in\{0,1,2, \ldots\}$ and the poles of $\Gamma(1-s)$, which are given by $s=k+1$ where $k \in\{0,1,2, \ldots\}$. 
Note that the first set of poles lies to the left of the integration path while the other two sets lie to its right. Therefore, we should consider either the first set alone or the second and third ones alone. The choice of poles depends on the values of $\bar{\gamma}$.

For very high values of $\bar{\gamma}(\bar{\gamma} \rightarrow \infty)$, we require to have positive powers of $C$ in the asymptotic expansion of $\bar{P}_{m}$ since $C$ is inversely proportional to $\bar{\gamma}$. It can be conjectured that the required expansion is obtained by evaluating the residues of the integral in (15) at the first set of poles and a formal proof of this statement is given in [23] ${ }^{1}$. First, let us consider the simple scenario where all the poles of the first set are simple, i.e., $\left(b_{j_{1}}+k_{1}\right) / B_{j_{1}} \neq\left(b_{j_{2}}+k_{2}\right) / B_{j_{2}}$ for $j_{1} \neq j_{2}$ and any non-negative integers $k_{1}, k_{2}$. For $H$-functions with $m=1$, this is obviously guaranteed. Fortunately, this is the case for the vast majority of fading distributions of interest. Thus, the asymptotic expansion is given by

$$
\bar{P}_{m} \sim \mathcal{K}\left(\frac{\lambda}{2}\right)^{u} \sum_{j=1}^{m} \sum_{k=0}^{\infty} \frac{\Gamma\left(1+\frac{b_{j}+k}{B_{j}}\right)}{\Gamma(u+1)}{ }_{1} F_{1}\left(-\frac{b_{j}+k}{B_{j}}+u-1 ; u+1 ;-\frac{\lambda}{2}\right) e_{j, k} C^{\frac{b_{j}+k}{B_{j}}},
$$

where the constants $e_{j, k}$ are given by

$$
e_{j, k}=\operatorname{Res}\left(\Xi(s),-\frac{b_{j}+k}{B_{j}}\right)=\frac{(-1)^{k}}{k ! B_{j}} \frac{\prod_{\substack{j^{\prime}=1 \\ j^{\prime} \neq j}}^{m} \Gamma\left(b_{j^{\prime}}-B_{j^{\prime}} \frac{b_{j}+k}{B_{j}}\right) \prod_{j^{\prime}=1}^{n} \Gamma\left(1-a_{j^{\prime}}+A_{j^{\prime}} \frac{b_{j}+k}{B_{j}}\right)}{\prod_{j^{\prime}=m+1}^{q} \Gamma\left(1-b_{j^{\prime}}+B_{j^{\prime}} \frac{b_{j}+k}{B_{j}}\right) \prod_{j^{\prime}=n+1}^{p} \Gamma\left(a_{j^{\prime}}-A_{j^{\prime}} \frac{b_{j}+k}{B_{j}}\right)} .
$$

Practically, for very high average SNR, e.g., $\bar{\gamma} \geq 20 \mathrm{~dB}$, the first few terms of the series w.r.t. $k$ dominate the sum yielding the following simplified expression.

$$
\bar{P}_{m} \sim \mathcal{K}\left(\frac{\lambda}{2}\right)^{u} \sum_{j=1}^{m} \sum_{k=0}^{K-1} \frac{\Gamma\left(1+\frac{b_{j}+k}{B_{j}}\right)}{\Gamma(u+1)}{ }_{1} F_{1}\left(-\frac{b_{j}+k}{B_{j}}+u-1 ; u+1 ;-\frac{\lambda}{2}\right) \frac{e_{j, k}}{B_{j}} C^{\frac{b_{j}+k}{B_{j}}},
$$

where typically $K=2$ when $\bar{\gamma} \geq 20 \mathrm{~dB}$.

For the case of $H$-functions with $m>1$, there is a possibility that poles from different Gamma factors coincide yielding multiple poles. We already considered a similar situation for another problem in [16]. Due to space limitations, we state the asymptotic expansion of $\bar{P}_{m}$ for this case in the following theorem without a proof (for more details, refer to [16, Theorem 2] or [23]).

\footnotetext{
${ }^{1}$ In fact, asymptotic expansions are given only for the $H$-function in [23]. However, it can be shown that the confluent hypergeometric function does not influence the asymptotic behavior because it grows much slower than the gamma functions in $\Xi(s)$ as $|s| \rightarrow \infty$.
} 
Theorem 2. Consider the $H$-function and define $s_{j, k} \equiv-\left(b_{j}+k\right) / B_{j}$ where $j=1, \ldots, m$ and $k \in\{0,1,2, \ldots\}$. Let $S$ be the unique set of the poles $s_{j, k}$. For each unique pole $s_{j, k} \in S$, define the sets of indexes $L\left(s_{j, k}\right)=\left\{l: s_{l, r}=s_{j, k}, l \in\{1, \ldots, m\}, r \in\{0,1,2, \ldots\}\right\}$ and $R\left(s_{j, k}\right)=\left\{r: s_{l, r}=s_{j, k}, l \in\{1, \ldots, m\}, r \in\{0,1,2, \ldots\}\right\}$ and let $N\left(s_{j, k}\right)=\left|L\left(s_{j, k}\right)\right|$ be the multiplicity of the pole $s_{j, k}$. Then, the asymptotic expansion of $\bar{P}_{m}$ is given by

$$
\bar{P}_{m} \sim \mathcal{K}\left(\frac{\lambda}{2}\right)^{u} \sum_{s_{j, k} \in S} \frac{\Gamma\left(1-s_{j, k}\right)}{\Gamma(u+1)}{ }_{1} F_{1}\left(s_{j, k}+u-1 ; u+1 ;-\frac{\lambda}{2}\right) e\left(s_{j, k}\right)[\log (C)]^{N\left(s_{j, k}\right)-1} C^{-s_{j, k}},
$$

where

$$
e\left(s_{j, k}\right)=\frac{(-1)^{N\left(s_{j, k}\right)-1}}{N\left(s_{j, k}\right) !} \frac{\prod_{r \in R\left(s_{j, k}\right)} \frac{(-1)^{r}}{r !}}{\prod_{l \in L\left(s_{j, k}\right)} B_{l}} \frac{\left.\prod_{j^{\prime} \notin L\left(s_{j, k}\right)}^{q} \Gamma\left(b_{j^{\prime}}+B_{j^{\prime}} s_{j, k}\right) \prod_{j^{\prime}=1}^{n} \Gamma\left(1-a_{j^{\prime}}-A_{j^{\prime}} s_{j, k}\right)\right]}{\prod_{j^{\prime}=m+1}^{q} \Gamma\left(1-b_{j^{\prime}}-B_{j^{\prime}} s_{j, k}\right) \prod_{j^{\prime}=n+1}^{p} \Gamma\left(a_{j^{\prime}}+A_{j^{\prime}} s_{j, k}\right)} .
$$

The asymptotic expansions for the common distributions treated in Table I are shown in Table II. It is evident that the given expressions are very easy to compute and do not require the evaluation of a numerical integration. Moreover, when the false alarm probability is very close to either 0 or 1 , simpler relations between $\bar{P}_{m}$ and $\lambda$ can be established. This is achieved by further approximating the confluent hypergeometric functions in the given expressions for very small values of $\lambda$ (when $P_{f}$ is close to 1 ) or very large values of $\lambda$ (when $P_{f}$ is close to 0 ).

The case of very small $\bar{\gamma}(\bar{\gamma} \rightarrow 0)$ is also of interest because it is important to be able to detect the presence of the primary user (PU) even in very low SNRs. In this case, $\bar{P}_{m}$ is obtained by evaluating the residues at the poles on the right of the integration path. Generally, the asymptotic expansion is given by

$$
\bar{P}_{m} \sim-\mathcal{K} \frac{\left(\frac{\lambda}{2}\right)^{u}}{\Gamma(u+1)} \sum_{s^{\prime} \in S}{ }_{1} F_{1}\left(u+s^{\prime}-1 ; u+1 ;-\frac{\lambda}{2}\right) \operatorname{Res}\left(\Xi(s) \Gamma(1-s), s^{\prime}\right) C^{-s^{\prime}},
$$

where $S=\{1,2, \ldots\} \cup\left\{\frac{k+1-a_{j}}{A_{j}}\right\}_{j=1, \ldots, n, k=0,1, \ldots}$ and the minus sign is because the contour of integration is circling the poles in the clock-wise direction. Again, there is a possibility that some poles are not simple, which may complicate the final expressions. Fortunately, in all the fading distributions of interest, we have $n=0$, i.e., there are no $\Gamma\left(1-a_{j}-A_{j} s\right)$ factors. Hence, we are left only with the poles of the factor $\Gamma(1-s)$. In this case, the asymptotic expansion 
TABLE II

ASYMPTOTIC EXPANSIONS OF $\bar{P}_{m}$ FOR DIFFERENT SPECIAL CASES OF THE FoX's $H$ - FADING DistRIBUTION WHEN $\bar{\gamma} \rightarrow \infty$.

\begin{tabular}{c|l}
\hline \hline Fading distribution & Asymptotic expression for $\bar{P}_{m}$ \\
\hline \hline Rayleigh & $\bar{P}_{m} \sim \frac{\left(\frac{\lambda}{2}\right)^{u}}{\Gamma(u+1)} \sum_{k=0}^{K-1}(-1)^{k}{ }_{1} F_{1}\left(u-k-1 ; u+1 ;-\frac{\lambda}{2}\right) \bar{\gamma}^{-k-1}$ \\
\hline Maxwell & $\bar{P}_{m} \sim \frac{2}{\sqrt{\pi} \frac{\left(\frac{\lambda}{2}\right)^{u}}{\Gamma(u+1)} \sum_{k=0}^{K-1} \frac{(-1)^{k} \Gamma\left(k+\frac{3}{2}\right)}{k !}{ }_{1} F_{1}\left(u-k-\frac{3}{2} ; u+1 ;-\frac{\lambda}{2}\right)\left(\frac{2 \bar{\gamma}}{3}\right)^{-k-\frac{3}{2}}}$ \\
\hline Nakagami-m & $\bar{P}_{m} \sim \frac{1}{\Gamma(m)} \frac{\left(\frac{\lambda}{2}\right)^{u}}{\Gamma(u+1)} \sum_{k=0}^{K-1} \frac{(-1)^{k} \Gamma(k+m)}{k !}{ }_{1} F_{1}\left(u-k-m ; u+1 ;-\frac{\lambda}{2}\right)\left(\frac{\bar{\gamma}}{m}\right)^{-k-m}$ \\
\hline Weibull & $\bar{P}_{m} \sim \alpha \frac{\left(\frac{\lambda}{2}\right)^{u}}{\Gamma(u+1)} \sum_{k=0}^{K-1} \frac{(-1)^{k} \Gamma(\alpha k+\alpha)}{k !}{ }_{1} F_{1}\left(u-\alpha k-\alpha ; u+1 ;-\frac{\lambda}{2}\right)\left(\frac{\bar{\gamma}}{\beta}\right)^{-\alpha k-\alpha}$ \\
\hline EGK & $\bar{P}_{m} \sim \frac{\xi}{\Gamma(\mu)} \frac{\left(\frac{\lambda}{2}\right)^{u}}{\Gamma(u+1)} \sum_{k=0}^{K-1} \frac{(-1)^{k} \Gamma(\xi k+\xi \mu)}{k !}{ }_{1} F_{1}\left(u-\xi k-\xi \mu ; u+1 ;-\frac{\lambda}{2}\right)\left(\frac{\bar{\gamma}}{\beta}\right)^{-\xi k-\xi \mu}$ \\
& $\sum_{m} \sim \frac{1}{\Gamma(\mu) \Gamma\left(\mu_{s}\right)} \frac{\left(\frac{\lambda}{2}\right)^{u}}{\Gamma(u+1)} \times$ \\
& $\begin{array}{c}\sum_{j, k} \in S \\
\text { where } S=\left\{\xi_{j}\left(\mu_{j}+k\right): j \in\{1,2\}, k \in\{0,1, \ldots, K-1\}\right\}, \mu_{1}=\mu, \xi_{1}=\xi, \mu_{2}=\mu_{s}, \xi_{2}= \\
\xi_{s}, \text { and } e\left(s_{j, k}\right) \text { is given by }(20) .\end{array}$ \\
\hline \hline
\end{tabular}

greatly simplifies to

$$
\bar{P}_{m} \sim \mathcal{K} \frac{\left(\frac{\lambda}{2}\right)^{u}}{\Gamma(u+1)} \sum_{k=1}^{\infty}{ }_{1} F_{1}\left(u+k-1 ; u+1 ;-\frac{\lambda}{2}\right) \Xi(k) \frac{(-1)^{k-1}}{(k-1) !} C^{-k} .
$$

Similar to the case of high $\bar{\gamma}$, the first few terms dominate the above sum when $\bar{\gamma}$ is very small (generally below $0 \mathrm{~dB}$ ). Based on our observations, when $\bar{\gamma}$ is extremely low, we may set $K=2$ in the previous expression, which will result in the following interesting expression:

$$
\bar{P}_{m} \sim \frac{\left(\frac{\lambda}{2}\right)^{u}}{\Gamma(u+1)}\left[{ }_{1} F_{1}\left(u ; u+1 ;-\frac{\lambda}{2}\right)-e^{-\lambda / 2} \bar{\gamma}\right],
$$

where we used $\mathcal{K} \Xi(1) C^{-1}=f^{*}(1)=1, \mathcal{K} \Xi(2) C^{-2}=f^{*}(2)=\bar{\gamma}$, and ${ }_{1} F_{1}(a, a, z)=e^{z}$. That is, the performance of the detection system will be almost independent of the fading distribution if $\bar{\gamma}$ is extremely low, which is quite expected. The general asymptotic expressions for low $\bar{\gamma}$ are given in Table III. 
TABLE III

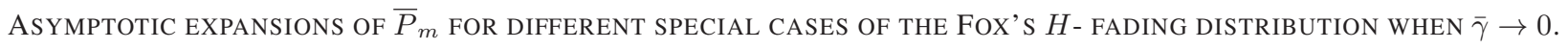

\begin{tabular}{c|c}
\hline \hline Fading distribution & Asymptotic expression for $\bar{P}_{m}$ \\
\hline \hline Rayleigh & $\bar{P}_{m} \sim \frac{\left(\frac{\lambda}{2}\right)^{u}}{\Gamma(u+1)} \sum_{k=1}^{K}{ }_{1} F_{1}\left(u+k-1 ; u+1 ;-\frac{\lambda}{2}\right)(-1)^{k-1} \bar{\gamma}^{k-1}$ \\
\hline Maxwell & $\bar{P}_{m} \sim \frac{2}{\sqrt{\pi}} \frac{\left(\frac{\lambda}{2}\right)^{u}}{\Gamma(u+1)} \sum_{k=1}^{K}{ }_{1} F_{1}\left(u+k-1 ; u+1 ;-\frac{\lambda}{2}\right) \Gamma\left(k+\frac{1}{2}\right) \frac{(-1)^{k-1}}{(k-1) !}\left(\frac{2 \bar{\gamma}}{3}\right)^{k-1}$ \\
\hline Nakagami- $m$ & $\bar{P}_{m} \sim \frac{1}{\Gamma(m)} \frac{\left(\frac{\lambda}{2}\right)^{u}}{\Gamma(u+1)} \sum_{k=1}^{K}{ }_{1} F_{1}\left(u+k-1 ; u+1 ;-\frac{\lambda}{2}\right) \Gamma(k+m-1) \frac{(-1)^{k-1}}{(k-1) !}\left(\frac{\bar{\gamma}}{m}\right)^{k-1}$ \\
\hline Weibull & $\bar{P}_{m} \sim \frac{\left(\frac{\lambda}{2}\right)^{u}}{\Gamma(u+1)} \sum_{k=1}^{K}{ }_{1} F_{1}\left(u+k-1 ; u+1 ;-\frac{\lambda}{2}\right) \Gamma\left(\frac{k}{\alpha}+1-\frac{1}{\alpha}\right) \frac{(-1)^{k-1}}{(k-1) !}\left(\frac{\bar{\gamma}}{\beta}\right)^{k-1}$ \\
\hline Generalised Gamma & $\bar{P}_{m} \sim \frac{1}{\Gamma(\mu)} \frac{\left(\frac{\lambda}{2}\right)^{u}}{\Gamma(u+1)} \sum_{k=1}^{K}{ }_{1} F_{1}\left(u+k-1 ; u+1 ;-\frac{\lambda}{2}\right) \Gamma\left(\frac{k}{\xi}+\mu-\frac{1}{\xi}\right) \frac{(-1)^{k-1}}{(k-1) !}\left(\frac{\bar{\gamma}}{\beta}\right)^{k-1}$ \\
\hline EGK & $\bar{P}_{m} \sim \frac{1}{\Gamma(\mu) \Gamma\left(\mu_{s}\right)} \frac{\left(\frac{\lambda}{2}\right)^{u}}{\Gamma(u+1)} \times$ \\
& $\sum_{k=1}^{K}{ }_{1} F_{1}\left(u+k-1 ; u+1 ;-\frac{\lambda}{2}\right) \Gamma\left(\mu-\frac{1}{\xi}+\frac{k}{\xi}\right) \Gamma\left(\mu_{s}-\frac{1}{\xi_{s}}+\frac{k}{\xi_{s}}\right) \frac{(-1)^{k-1}}{(k-1) !}\left(\frac{\bar{\gamma}}{\beta \beta_{s}}\right)^{k-1}$ \\
\hline \hline
\end{tabular}

\section{NumericAl RESUlts}

In this section, we verify the accuracy of our derived expressions. In all figures, the reference values of $\bar{P}_{m}$ were obtained by numerically evaluating the integration in (1) (after subtracting from one) using 5000 sample values of the integrand and 100 points for the computation of the $H$-function whenever necessary. The values of our expression were computed using the Python implementation of the multivariate- $H$ function in [16] and it is based on a grid of 2500 sample points $(50 \times 50)$. The number of points was chosen such that both methods provide similar accuracy. The reference values in all cases are shown as solid curves whereas the values of our expressions are denoted by markers.

Fig. 1 shows the complementary receiver operating characteristics (ROC) for various simple fading distributions with $u=1.5$ and $\bar{\gamma}=10 \mathrm{~dB}$. It is clear from the figure that the derived closed-form expression in (14) is indistinguishable from the result obtained via the integration in (1). It is worth mentioning here that our derived expression is calculated much faster than the numerical integration. Specifically, on a $3 \mathrm{GHz}$, quad-core, 8 GB RAM laptop, the average computation time of the numerical integration method was 7.98 seconds per point versus merely 0.038 seconds for our derived expression. This amounts to a speed factor of approximately 209 


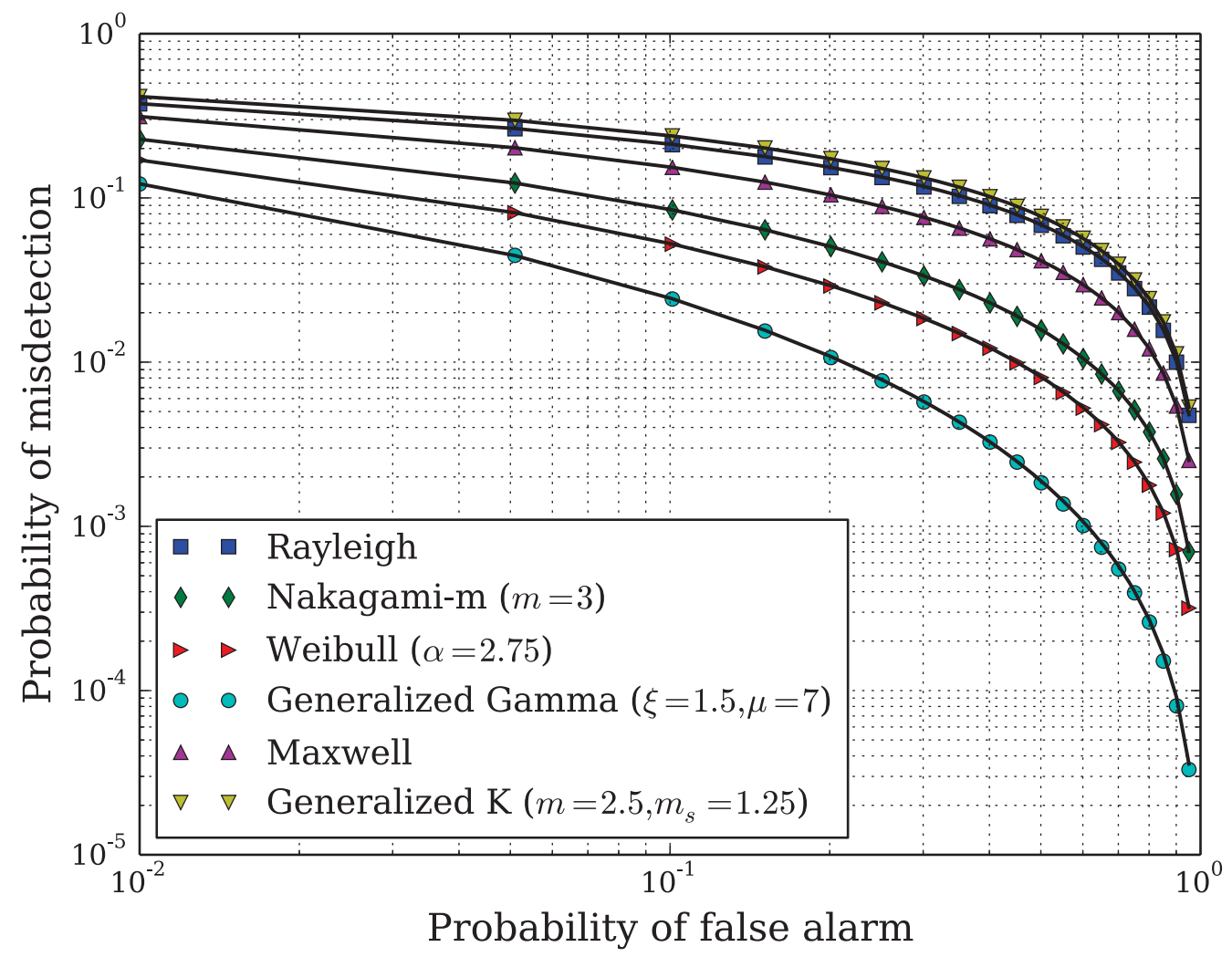

Fig. 1. Exact complementary ROC curves for different simple fading distributions with $u=1.5$ and $\bar{\gamma}=10 \mathrm{~dB}$. Solid lines represent numerical integration while the markers represent the novel derived expression.

without sacrificing the accuracy.

Figs. 2 and 3 show both the exact $\bar{P}_{m}$ from (1) with $\bar{\gamma}=20 \mathrm{~dB}$ as well as the high-SNR asymptotic expansion in (18) for simple and composite fading distributions with $u=5$ and $u=1.5$, respectively. Note that the case of colliding poles occurs in the Weibull-Exponential fading distribution only. The results show that the high-SNR asymptotic expansion matches very well the exact expression and they are also hardly distinguishable from each other.

As for the behavior of the low-SNR asymptotic expansion, we choose to investigate this over the two Fox-Cox models used in [14, Table IV] (See Table IV). These models were obtained via curve fitting to real measurements of scaled multipath fading of a $5.2 \mathrm{GHz}$ band $\mathrm{V} 2 \mathrm{~V}$ channel and they correspond to $\bar{\gamma}$ of about $-4 \mathrm{~dB}$, which makes them a very good candidate to test our proposed low-SNR asymptotic expansion. Fig. 4 shows the exact and asymptotic expansion for 


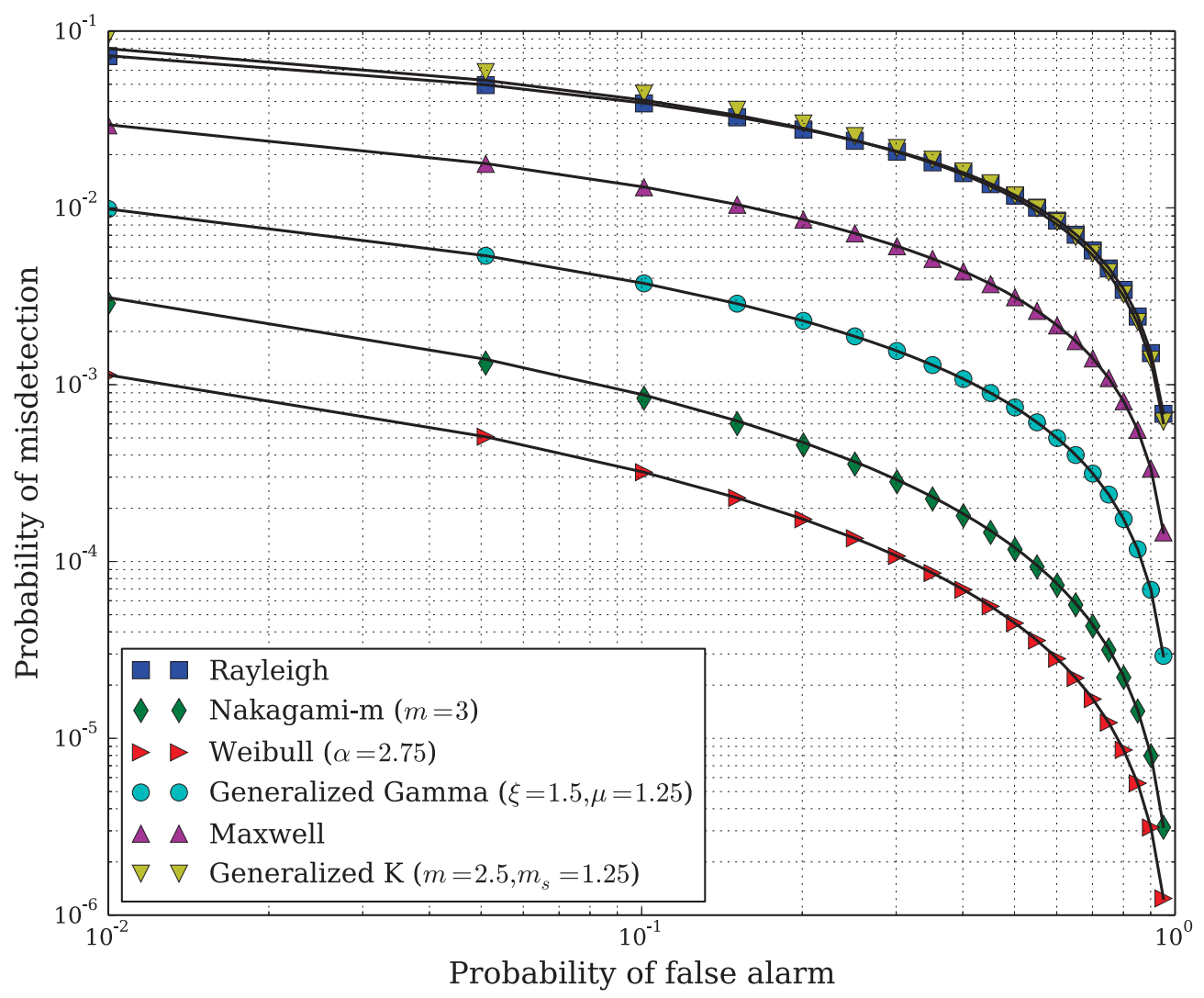

Fig. 2. Exact and high-SNR asymptotic complementary ROC curves for different simple fading distributions with $u=5$ and $\bar{\gamma}=20 \mathrm{~dB}$.

TABLE IV

FOX $H$-FITS TO MEASUREMENTS FOR SCALED MULTI-PATH FADING IN V2V COMMUNICATION.

\begin{tabular}{c|cccc|cccc}
\hline \hline & $m$ & $n$ & $p$ & $q$ & $\mathcal{K}$ & $C$ & $\left\{\left(a_{j}, A_{j}\right)\right\}$ & $\left\{\left(b_{j}, B_{j}\right)\right\}$ \\
\hline \hline Measurements 1 & 3 & 0 & 0 & 3 & 0.225 & 5.774 & - & $\{(1.5,0.5),(0.4,0.5),(4.5,0.5)\}$ \\
\hline Measurements 2 & 3 & 0 & 0 & 3 & 2.874 & 3.940 & - & $\{(0.45,0.5),(2,0.5),(1.8,0.2)\}$ \\
\hline \hline
\end{tabular}

different choices of $u$. It is clear that they indeed match well over a significant portion of the ROC curve. Moreover, the ROC curves for different fading channels get closer to each other, which is consistent with our observation near the end of Section IV. 


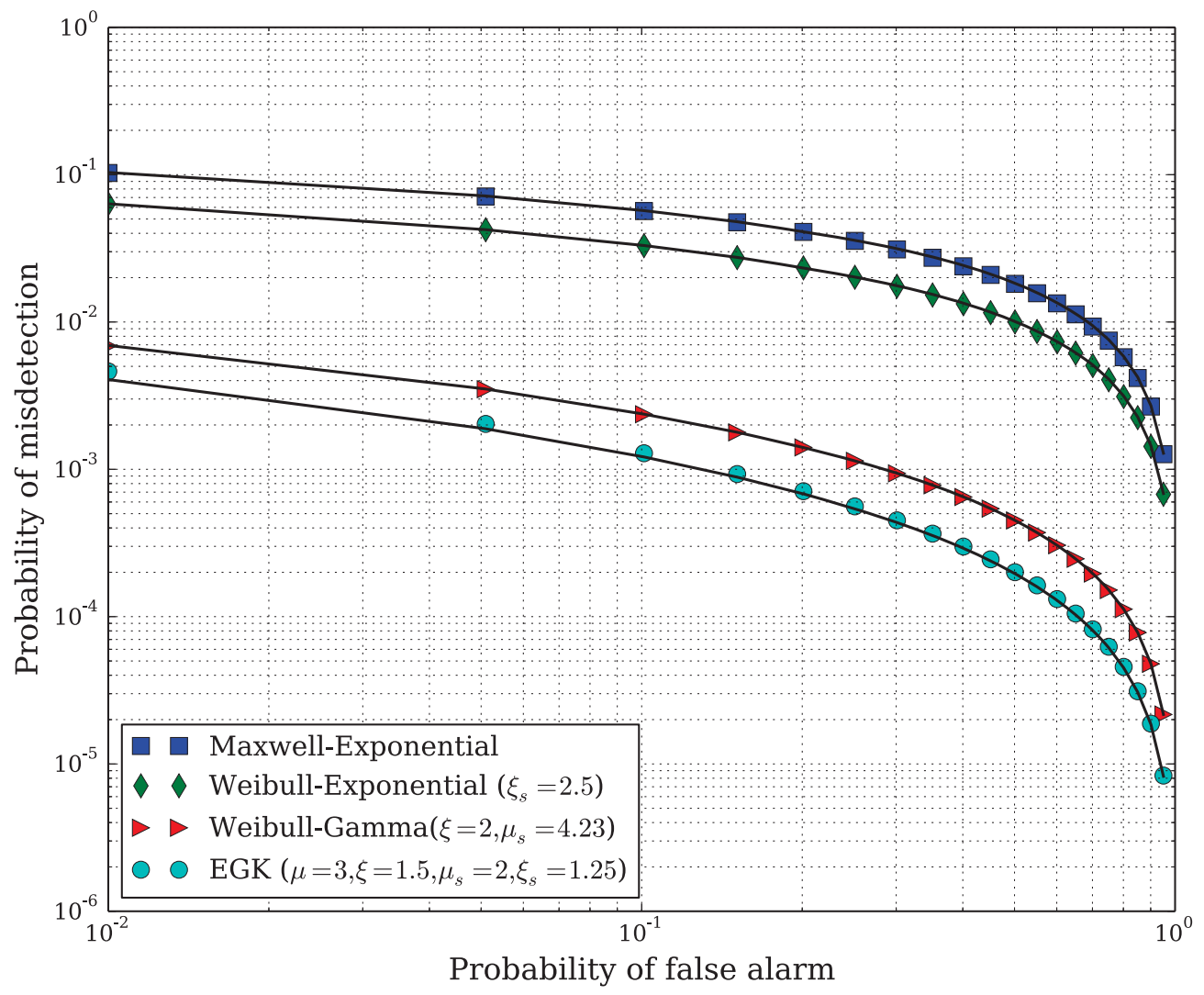

Fig. 3. Exact and high-SNR asymptotic complementary ROC curves for different composite fading distributions with $u=1.5$ and $\bar{\gamma}=20 \mathrm{~dB}$.

\section{CONCLUSION}

We presented a Mellin transform based unified approach for calculating the probability of misdetection of the PU in a cognitive radio network. We then applied this approach to the very general case of $H$-function fading and obtained a closed-form expression for the probability of detection in terms of the bivariate $H$-function. The obtained expression was shown to reduce to those previously obtained in the literature for Nakagami- $m$ and EGK fading and the proposed approach has been shown to be a powerful and fast tool for performance evaluation over different fading channels. We also proposed low- and high-SNR asymptotic expansions that are much simpler to calculate and were shown to provide very good approximation for $\bar{P}_{m}$. Moreover, they can be easily used for comparing the performance over different fading channels for high 


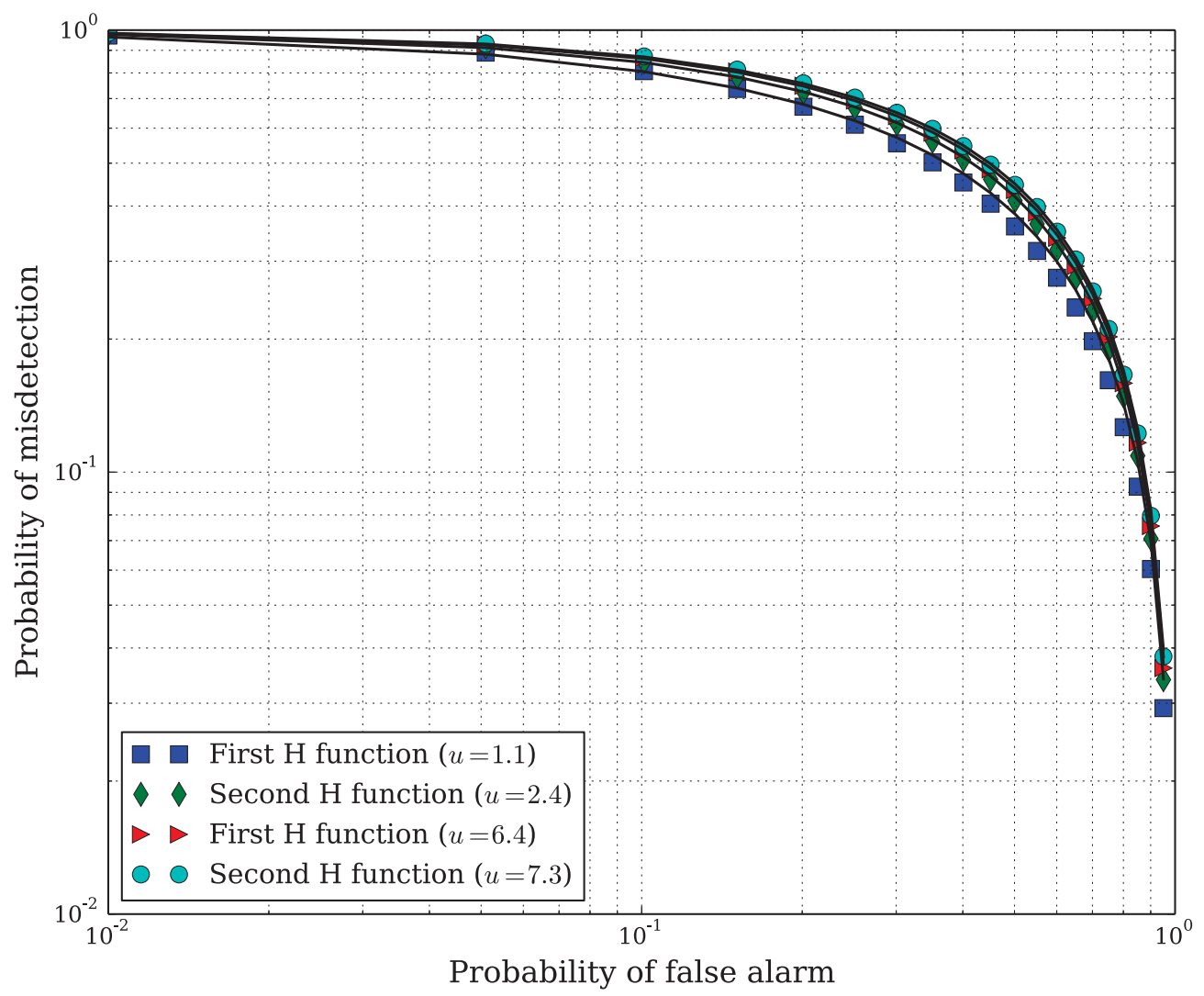

Fig. 4. Exact and low-SNR asymptotic complementary ROC curves for the Fox-Cox model in [14, Table IV] with various values of $u$.

average SNRs.

Though there are some fading distributions that are not special cases of the Fox's $H$-function such as the Rice and the log-normal distributions, we believe that the unified framework we developed in Section II will still be applicable to those cases. The main difference, however, is that the obtained expressions will be in terms of the tri-variate Fox's $H$-function instead.

\section{APPENDIX A}

\section{THE NAKAGAMI- $m$ FADING DISTRIBUTION}

The representation of the Nakagami- $m$ distribution as an $H$-function as well as the corresponding expression for $\bar{P}_{m}$ are shown in Table I. It can be shown that this expression is equivalent 
to that in [1, Eq. (20)] for integer $u$ by first proving the following lemma.

Lemma 1. For the Nakagami-m distribution, we have

$$
\bar{P}_{m}(u)=\bar{P}_{m}(u-1)-\left(1+\frac{\bar{\gamma}}{m}\right)^{-m} e^{-\lambda / 2} \frac{(\lambda / 2)^{u-1}}{\Gamma(u)}{ }_{1} F_{1}\left(m ; u ; \frac{\lambda \bar{\gamma}}{2(m+\bar{\gamma})}\right),
$$

where $\bar{P}_{m}(u)$ is the average probability of misdetection at a certain time-bandwidth product $u$ and $u>1$.

Proof. Using the definition of the multivariate $H$-function, it is straightforward to show that

$$
\begin{aligned}
\bar{P}_{m} & =\frac{(\lambda / 2)^{u}}{\Gamma(m)(2 \pi i)^{2}} \iint \frac{\Gamma(-s) \Gamma(-w) \Gamma(u+s+w) \Gamma(m+s)}{\Gamma(u+s) \Gamma(u+1+w)}\left(\frac{\lambda}{2}\right)^{w}\left(\frac{\bar{\gamma}}{m}\right)^{s} d s d w \\
& =\frac{(\lambda / 2)^{u}}{\Gamma(m)(2 \pi i)} \int \frac{\Gamma(-s) \Gamma(m+s)}{\Gamma(u+1)}{ }_{1} F_{1}\left(u+s ; u+1 ;-\frac{\lambda}{2}\right)\left(\frac{\bar{\gamma}}{m}\right)^{s} d s .
\end{aligned}
$$

With the aid of [19, Eq. (9.212.2)] and performing some algebraic manipulations, we can show that

$$
\frac{(\lambda / 2)^{u}}{\Gamma(u+1)}{ }_{1} F_{1}\left(u+s ; u+1 ;-\frac{\lambda}{2}\right)=\frac{(\lambda / 2)^{u-1}}{\Gamma(u)}\left[{ }_{1} F_{1}\left(u-1+s ; u ;-\frac{\lambda}{2}\right)-{ }_{1} F_{1}\left(u+s ; u ;-\frac{\lambda}{2}\right)\right] .
$$

Substituting (26) into (25), we can prove that

$$
\begin{aligned}
P_{m}(u) & =P_{m}(u-1)-\frac{(\lambda / 2)^{u-1}}{\Gamma(m)(2 \pi i)} \int \frac{\Gamma(-s) \Gamma(m+s)}{\Gamma(u)}{ }_{1} F_{1}\left(u+s ; u ;-\frac{\lambda}{2}\right)\left(\frac{\bar{\gamma}}{m}\right)^{s} d s \\
& =P_{m}(u-1)-\frac{(\lambda / 2)^{u-1} e^{-\lambda / 2}}{\Gamma(m)(2 \pi i)} \int \frac{\Gamma(-s) \Gamma(m+s)}{\Gamma(u)}{ }_{1} F_{1}\left(-s ; u ; \frac{\lambda}{2}\right)\left(\frac{\bar{\gamma}}{m}\right)^{s} d s,
\end{aligned}
$$

in which we used the Kummer transformation of the confluent hypergeometric function in deriving the last line. Substituting ${ }_{1} F_{1}\left(-s ; u ; \frac{\lambda}{2}\right)$ by its Mellin-integral representation, we get

$$
\begin{aligned}
P_{m}(u) & =P_{m}(u-1)-\frac{(\lambda / 2)^{u-1} e^{-\lambda / 2}}{\Gamma(m)(2 \pi i)^{2}} \iint \frac{\Gamma(-w) \Gamma(w-s) \Gamma(m+s)}{\Gamma(u+w)}\left(-\frac{\lambda}{2}\right)^{w}\left(\frac{\bar{\gamma}}{m}\right)^{s} d s d w \\
& =P_{m}(u-1)-\frac{(\lambda / 2)^{u-1} e^{-\lambda / 2}}{\Gamma(m)(2 \pi i)} \int \frac{\Gamma(-w)}{\Gamma(u+w)}\left(-\frac{\lambda}{2}\right)^{w}\left(\frac{1}{2 \pi i} \int \Gamma(w-s) \Gamma(m+s)\left(\frac{\bar{\gamma}}{m}\right)^{s} d s\right) d w .
\end{aligned}
$$

The inner integral between parenthesis in the previous expression is equal to

$$
\begin{aligned}
\frac{1}{2 \pi i} \int \Gamma(w-s) \Gamma(m+s)\left(\frac{\bar{\gamma}}{m}\right)^{s} d s & ={ }_{1} F_{0}\left(m+w ;-;-\frac{\bar{\gamma}}{m}\right) \Gamma(m+w)\left(\frac{\bar{\gamma}}{m}\right)^{w} \\
& =\Gamma(m+w)\left(1+\frac{\bar{\gamma}}{m}\right)^{-m-w}\left(\frac{\bar{\gamma}}{m}\right)^{w} .
\end{aligned}
$$


Substituting (29) into (28) and applying the Mellin-Barnes integral representation of the confluent hypergeometric function to the resultant expression, the lemma follows.

When $u$ is a positive integer, we can prove that $\bar{P}_{m}$ is given by the following theorem.

Theorem 3. If $u$ is a positive integer, then

$$
\bar{P}_{m}(u)=\bar{P}_{m}(1)-\left(1+\frac{\bar{\gamma}}{m}\right)^{-m} e^{-\lambda / 2} \sum_{k=0}^{u-1} \frac{(\lambda / 2)^{k}}{k !}{ }_{1} F_{1}\left(m ; k+1 ; \frac{\lambda \bar{\gamma}}{2(m+\bar{\gamma})}\right),
$$

where

$$
\bar{P}_{m}(1)=1-\frac{1}{\Gamma(m)}\left(\frac{m}{\bar{\gamma}}\right)^{m} \int_{\gamma=0}^{\infty} \gamma^{m-1} e^{-m \gamma / \bar{\gamma}} Q_{1}(\sqrt{2 \gamma}, \sqrt{\lambda}) d \gamma .
$$

Proof. By successive application of Lemma 1, we can easily show that for a positive integer $n \leq u-1$

$$
\bar{P}_{m}(u)=\bar{P}_{m}(u-n)-\left(1+\frac{\bar{\gamma}}{m}\right)^{-m} e^{-\lambda / 2} \sum_{k^{\prime}=1}^{n} \frac{(\lambda / 2)^{u-k^{\prime}}}{\Gamma\left(u-k^{\prime}+1\right)}{ }_{1} F_{1}\left(m ; u-k^{\prime}+1 ; \frac{\lambda \bar{\gamma}}{2(m+\bar{\gamma})}\right) .
$$

Since $u$ is a positive integer, we have $\Gamma\left(u-k^{\prime}+1\right)=\left(u-k^{\prime}\right)$ !. Setting $n=u-1$ and performing the change of variables $k=u-k^{\prime}$ in the previous expression, the first part of the theorem is proved. The expression of $\bar{P}_{m}(1)$ is proved by substituting in (3) with $P_{m}(\gamma)=$ $1-Q_{1}(\sqrt{2 \gamma}, \sqrt{\lambda})$.

The expression in (30) can be shown to exactly match that given in [1, Eq. (20)] after some straightforward manipulations.

\section{APPENDIX B}

\section{THE EGK DISTRIBUTION}

By substituting the EGK parameters (see Table I) in (14), we obtain the corresponding expression in Table I. An alternative form is given in [12] as

$$
\bar{P}_{m}=\sum_{n=0}^{\infty} \frac{\Gamma_{l}(n+u, \lambda / 2)}{n ! \Gamma(n+u)} \frac{1}{\Gamma\left(m_{s}\right) \Gamma(m)} H_{1,2}^{2,1}\left(\frac{\beta_{s} \beta}{\bar{\gamma}} \mid \begin{array}{c}
(1-n, 1) \\
\left(m_{s}, \frac{1}{\xi_{s}}\right),\left(m, \frac{1}{\xi}\right)
\end{array}\right),
$$

where $\Gamma_{l}(.,$.$) denotes the lower incomplete gamma function. A proof of the equivalency of the$ entry in Table I and (33) is as follows. Using the definition of the multivariate $H$-function and 
performing simple manipulations, we can prove that the entry in Table I reduces to

$$
\bar{P}_{m}=\frac{1}{2 \pi i} \int\left(\frac{\beta \beta_{s}}{\bar{\gamma}}\right)^{z} \Gamma\left(m-\frac{z}{\xi}\right) \Gamma\left(m_{s}-\frac{z}{\xi_{s}}\right) P_{m}^{*}(z) d z
$$

where $P_{m}^{*}(z)$ is given by Theorem 1 . In fact, $P_{m}^{*}(z)$ can also be expressed in terms of an infinite series as shown in the following theorem.

\section{Theorem 4.}

$$
P_{m}^{*}(z)=\sum_{n=0}^{\infty} \frac{\Gamma_{l}(n+u, \lambda / 2)}{n ! \Gamma(n+u)} \Gamma(z+n)
$$

where $\Gamma_{l}(.,$.$) is the lower incomplete gamma function.$

Proof. We start by deriving $P_{m}(\gamma)$, the inverse Mellin transform of $P_{m}^{*}(z)$. From (8a), we can deduce $P_{m}^{*}(z)$ has poles only at $z=0,-1,-2, \ldots$ since the confluent hypergeometric function is an entire function of its first argument. Hence, the inverse Mellin transform is simply given by

$$
P_{m}(\gamma)=\sum_{n=0}^{\infty} \operatorname{Res}\left(P_{m}^{*}(z) \gamma^{-z},-n\right)
$$

Substituting (36) into (5) and noting that $\operatorname{Res}(\Gamma(z),-n)=(-1)^{n} / n$ !, we obtain

$$
\begin{aligned}
P_{m}(\gamma) & =\sum_{n=0}^{\infty} \frac{(-\gamma)^{n}}{n ! \Gamma(u+n)} \frac{1}{2 \pi i} \int \frac{\Gamma(-w) \Gamma(u+n+w)}{\Gamma(u+1+w)}\left(\frac{\lambda}{2}\right)^{u+w} d w \\
& =\sum_{n=0}^{\infty} \frac{(-\gamma)^{n}}{n ! \Gamma(u+1)}\left(\frac{\lambda}{2}\right)^{u}{ }_{1} F_{1}\left(u+n ; u+1 ;-\frac{\lambda}{2}\right) .
\end{aligned}
$$

Using the relations [19, Eq. (9.212.1)] and [19, Eq. (8.972.1)], we can prove that

$\frac{1}{\Gamma(u+1)}{ }_{1} F_{1}\left(u+n ; u+1 ;-\frac{\lambda}{2}\right)=\frac{(n-1) ! e^{-\lambda / 2}}{\Gamma(u+n)} L_{n-1}^{u}\left(\frac{\lambda}{2}\right)=e^{-\lambda / 2} \sum_{k=0}^{n-1}(-1)^{k}\left(\begin{array}{c}n-1 \\ k\end{array}\right) \frac{(\lambda / 2)^{k}}{\Gamma(k+u+1)}$,

where $L_{n-1}^{u}($.$) is the associated Laguerre polynomial. Using the fact that e^{-\lambda / 2}(\lambda / 2)^{k+u}=$ $\Gamma_{l}(k+u+1, \lambda / 2)-(k+u) \Gamma_{l}(k+u, \lambda / 2)$ into (38), we can prove that

$$
\begin{aligned}
\frac{\left(\frac{\lambda}{2}\right)^{u}}{\Gamma(u+1)}{ }_{1} F_{1}\left(u+n ; u+1 ;-\frac{\lambda}{2}\right) & =\sum_{k=0}^{n-1}(-1)^{k}\left(\begin{array}{c}
n-1 \\
k
\end{array}\right)\left[\frac{\Gamma_{l}(k+u+1, \lambda / 2)}{\Gamma(k+u+1)}-\frac{\Gamma_{l}(k+u, \lambda / 2)}{\Gamma(k+u)}\right] \\
& =\sum_{k=0}^{n}(-1)^{k}\left(\begin{array}{l}
n \\
k
\end{array}\right) \frac{\Gamma_{l}(k+u, \lambda / 2)}{\Gamma(k+u)}
\end{aligned}
$$


The last line follows using some simple algebraic manipulations and using the Pascal's rule for binomial coefficients [24]. Substituting (39) into (37), we can now show that

$$
P_{m}(\gamma)=\sum_{n=0}^{\infty} \frac{(-\gamma)^{n}}{n !} \sum_{k=0}^{n}(-1)^{k}\left(\begin{array}{l}
n \\
k
\end{array}\right) \frac{\Gamma_{l}(k+u, \lambda / 2)}{\Gamma(k+u)} .
$$

Performing the change of variables $n=k+m, k=k$, we obtain

$$
P_{m}(\gamma)=\sum_{m=0}^{\infty} \frac{(-\gamma)^{m}}{m !} \sum_{k=0}^{\infty} \frac{\Gamma_{l}(k+u, \lambda / 2)}{\Gamma(k+u)} \frac{\gamma^{k}}{k !}=e^{-\gamma} \sum_{k=0}^{\infty} \frac{\Gamma_{l}(k+u, \lambda / 2)}{\Gamma(k+u)} \frac{\gamma^{k}}{k !} .
$$

Taking the Mellin transform of the last line the Theorem is proved.

Hence, upon substituting (35) into (34), we have

$$
\begin{aligned}
\bar{P}_{m} & =\frac{1}{2 \pi i} \int\left(\frac{\beta \beta_{s}}{\bar{\gamma}}\right)^{z} \Gamma\left(m-\frac{z}{\xi}\right) \Gamma\left(m_{s}-\frac{z}{\xi_{s}}\right) \sum_{n=0}^{\infty} \frac{\Gamma_{l}(n+u, \lambda / 2)}{n ! \Gamma(n+u)} \Gamma(z+n) d z \\
& =\sum_{n=0}^{\infty} \frac{\Gamma_{l}(n+u, \lambda / 2)}{n ! \Gamma(n+u)} \frac{1}{2 \pi i} \int \Gamma(z+n) \Gamma\left(m-\frac{z}{\xi}\right) \Gamma\left(m_{s}-\frac{z}{\xi_{s}}\right)\left(\frac{\beta \beta_{s}}{\bar{\gamma}}\right)^{z} d s,
\end{aligned}
$$

where the interchange between the integral and the infinite summation in the last line is allowed due to the boundedness of the absolute integral. Substituting $z=-s$ in the last expression and employing the definition of the $H$-function, (33) immediately follows.

\section{REFERENCES}

1 Digham, F., Alouini, M.-S., and Simon, M. K.: 'On the energy detection of unknown signals over fading channels'. Proc. IEEE International Conference on Communications (ICC '03), 5, 2003, 3575-3579 vol.5.

2 Digham, F., Alouini, M.-S., and Simon, M. K.: 'On the Energy Detection of Unknown Signals Over Fading Channels', IEEE Transactions on Communications, 2007, 55, (1), pp. 21-24.

3 Sofotasios, P., Fikadu, M., Ho-Van, K., and Valkama, M.: 'Energy detection sensing of unknown signals over Weibull fading channels'. Proc. International Conference on Advanced Technologies for Communications (ATC), 2013, pp. 414-419.

4 Shahini, A., Bagheri, A., and Shahzadi, A.: 'A unified approach to performance analysis of energy detection with diversity receivers over Nakagami-m fading channels'. Proc. 2013 International Conference on Connected Vehicles and Expo (ICCVE), 2013, pp. 707-712. 
5 Herath, S., Rajatheva, N., and Tellambura, C.: 'Energy Detection of Unknown Signals in Fading and Diversity Reception', IEEE Transactions on Communications, 2011, 59, (9), pp. 2443-2453.

6 Annamalai, A and Olaluwe, A: 'On the energy detection of unknown signals in $\kappa-\mu$ and $\eta$ - $\mu$; fading channels with diversity receivers'. Proc. International Conference on Connected Vehicles and Expo (ICCVE), 2013, pp. 127-132.

7 Rasheed, H., Haroon, F., and Adachi, F.: 'On the Energy Detection over generalized-K fading'. Proc. IEEE 14th International Multitopic Conference (INMIC), 2011, pp. 367-371.

8 Atapattu, S., Tellambura, C., and Jiang, H.: 'Performance of an Energy Detector over Channels with Both Multipath Fading and Shadowing', IEEE Transactions on Wireless Communications, 2010, 9, (12), pp. 3662-3670.

9 Fathi, Y. and Tawfik, M.: 'Versatile performance expression for energy detector over $\alpha-\mu$ generalised fading channels', Electronics Letters, 2012, 48, (17), pp. 1081-1082.

10 Sofotasios, P., Rebeiz, E., Zhang, L., Tsiftsis, T., Cabric, D., and Freear, S.: 'Energy Detection Based Spectrum Sensing Over $\kappa-\mu$ and $\kappa-\mu$ Extreme Fading Channels', IEEE Transactions on Vehicular Technology, 2013, 62, (3), pp. 1031-1040.

11 Atapattu, S., Tellambura, C., and Jiang, H.: 'Energy detection of primary signals over $\eta$ $\mu$; fading channels'. Proc. International Conference on Industrial and Information Systems (ICIIS), 2009, pp. 118-122.

12 Alhennawi, H., Ismail, M. H., and Mourad, H.-A.: 'Performance evaluation of energy detection over extended generalised- K composite fading channels', Electronics Letters, 2014, 50, (22), pp. 1643-1645.

13 Yilmaz, F. and Alouini, M.-S.: 'A Novel Unified Expression for the Capacity and Bit Error Probability of Wireless Communication Systems over Generalized Fading Channels', IEEE Trans. Commun., 2012, 60, (7), pp. 1862-1876.

14 Jeong, Y., Chong, J. W., Shin, H., and Win, M.: 'Intervehicle Communication: Cox-Fox Modeling', IEEE Journal on Selected Areas in Communications, 2013, 31, (9), pp. 418-433.

15 El Ayadi, M. M. H. and Ismail, M. H.: 'Novel Closed-Form Exact Expressions and Asymptotic Analysis for the Symbol Error Rate of Single and Multiple-Branch MRC and EGC Receivers over $\alpha-\mu$ Fading', IEEE Transactions on Vehicular Technology, 2014, PP, (99), pp. 1-1. 
16 Alhennawi, H. R., El Ayadi, M. M. H., Ismail, M. H., and Mourad, H.-A.: 'Closed-form Exact and Asymptotic Expressions for the Symbol Error Rate and Channel Capacity of the H-function Fading Channel', IEEE Transactions on Vehicular Technology, 2015, PP, (99), pp. $1-1$.

17 Simon, M. K. and Alouini, M.-S.: 'Digital Communication over Fading Channels' (John Wiley \& Sons, 2005, 1st edn.).

18 Berterand, J., Bertrand, P., and Ovarlez, J.-P.: 'The Transforms and Applications Handbook' (CRC Press, 1995, 1st edn.).

19 Gradshteyn, I. S. and Ryzhik, I. M.: 'Table of Integrals, Series and Products' (Academic Press, 2000, 1st edn.).

20 Olver, F. W. J., Lozier, D. W., Boisvert, R. F., and Clark, C. W., eds.: 'NIST Handbook of Mathematical Functions' (Cambridge University Press, 2010, 1st edn.).

21 Mathai, A., Saxena, R. K., and Haubold, H. J.: 'The $H$-function: Theory and Applications' (Springer, 2010, 1st edn.).

22 Peppas, K.: 'A New Formula for the Average Bit Error Probability of Dual-Hop Amplifyand-Forward Relaying Systems over Generalized Shadowed Fading Channels', IEEE Wireless Communications Letters, 2012, 1, (2), pp. 85-88.

23 Kilbas, A. and Siago, M.: 'H-Transforms Theory and Applications' (CRC Press LLC, 2004, 1 st edn.).

24 Merris, R.: 'Combinatorics', Wiley Series in Discrete Mathematics and Optimization (Wiley, 2003, 1st edn.). 